\title{
Bipolar Valued Fuzzy $\alpha$-Ideal of BF-Algebra
}

\author{
Shanmugavelu Sabarinathan', David C. Kumar'2, Prakasam Muralikrishna ${ }^{3}$ \\ ${ }^{1}$ Department of Mathematics, Latha Mathavan Engineering College, Madurai, India \\ ${ }^{2}$ Department of Mathematics, Vickram College of Engineering, Enathi, India \\ ${ }^{3} \mathrm{PG}$ and Research Department of Mathematics, Muthurangam Government Arts College (Autonomous), \\ Vellore, India \\ Email: sabarisiddha@gmail.com,dckumarmaths@gmail.com,pmkrishna@rocketmail.com
}

Received 24 May 2016; accepted 31 May 2016; published 19 August 2016

Copyright (C) 2016 by authors and Scientific Research Publishing Inc.

This work is licensed under the Creative Commons Attribution International License (CC BY).

http://creativecommons.org/licenses/by/4.0/

(c) (i) Open Access

\begin{abstract}
In the mathematical applications, ideal concepts are involved. They have been studied and analyzed in various ways. Already ideal and $\alpha$-ideal concepts were discussed in BF-algebras. In this paper the idea of bipolar valued fuzzy $\alpha$-ideal of BF algebra is proposed. The relationship between bipolar valued fuzzy ideal and bipolar valued fuzzy $\alpha$-ideal is studied. Some interesting results are also discussed.
\end{abstract}

\section{Keywords}

\section{BF-Algebra, Ideal, Bipolar Valued Fuzzy Ideal, Bipolar Valued Fuzzy $\alpha$-Ideal}

\section{Introduction}

After the concept of fuzzy sets of Zadeh [1], Lee [2] proposed an extension of fuzzy sets namely Bipolar Valued Fuzzy Sets (BVFS). Their range of membership degree has been extended from the interval $[0,1]$ to $[-1,1]$ and in [3]. He made a comparison with other fuzzy settings. These Bipolar valued fuzzy sets possess degrees of membership that denote the degree of satisfaction to the property corresponding to a fuzzy set and its counterproperty in a bipolar valued fuzzy set. The membership degree 0 refers that the elements are irrelevant to the corresponding property. Further, the membership degrees $(0,1]$ show that the elements somewhat satisfy the property, and the membership degrees $[-1,0)$ denote that the elements somewhat satisfy the implicit counter property.

There are two kinds of representations in the definition of bipolar valued fuzzy sets. They are canonical representation and reduced representation. In this work, the canonical representation of bipolar valued fuzzy sets is utilized.

In 2011, Bipolar valued fuzzy K-subalgebras are discussed by Farhat Nisar [4]. The authors of [5] studied the 
concepts of Intuitionistic L-fuzzy p-ideals of BF-algebras and their related results.

Inspired by the concepts recently, the concept of Filters of BCH-Algebras Based on Bipolar-Valued Fuzzy Sets [6] has been discussed. In this paper, these concepts are intended to $\alpha$-ideal of BF-algebras and bipolar valued fuzzy $\alpha$-ideal of a BF-algebra is proposed. The nature of the homomorphic images of bipolar valued fuzzy $\alpha$-ideal of a BF-algebra is also analyzed.

The paper is organized as follows: Section 2 provides the preliminaries. In Section 3, Bipolar valued fuzzy $\alpha$-ideal is discussed and in Section 4, homomorphism on Bipolar valued fuzzy $\alpha$-ideal is studied. Section 5 gives the conclusion.

\section{Preliminaries}

In this section, some basic definitions and results that are required in the sequel are recalled. The notations $\min (a, b)=a \wedge b$ and $\max (a, b)=a \vee b$ are used.

\subsection{Basic Results on BF-Algebras}

Definition 2.1. [7] A BF algebra is a non-empty set $X$ with a constant 0 and a single binary operation * which satisfies the following axioms:

- 1. $x * x=0$

- 2. $x * 0=x$

- 3. $0 *(x * y)=y * x$ for all $x, y \in X$.

Example 2.2. Let $X=\{0,1,2,3,4\}$ be a set which comprises the following table.

\begin{tabular}{llllll}
\hline$*$ & 0 & 1 & 2 & 3 & 4 \\
\hline 0 & 0 & 4 & 3 & 2 & 1 \\
1 & 1 & 0 & 4 & 3 & 2 \\
2 & 2 & 1 & 0 & 4 & 3 \\
3 & 3 & 2 & 1 & 0 & 4 \\
4 & 4 & 3 & 2 & 1 & 0 \\
\hline
\end{tabular}

Then $(X, *, 0)$ is BF-algebra.

Definition 2.3. [7] A BG-algebra is a non-empty set $X$ with a constant 0 and a single binary operation * satisfying the following axioms:

- $x * x=0$

- $x * 0=x$

- $(x * y) *(0 * y)=x$ for all $x, y \in X$.

A binary relation $\leq$ in a BF-algebra $X$ can be defined as $x \leq y$, if and only if $x * y=0$.

A subset $S$ of a BF-algebra $X$ is called a subalgebra of $X$, if $x * y \in S$ for all $x, y \in S$.

An ideal of a BF-algebra $X$ is a subset $I$ of $X$ consisting 0 such that, if $x * y \in I$ and $y \in I$, then $x \in I$.

An ideal $I$ of a BF-algebra $X$ is called closed, if $0 * x \in I \forall x \in I$.

A non-empty subset $I$ of a BF-algebra $X$ is $\alpha$-ideal, if for all $x, y, z \in X,(x * z) *(y * z) \in I$ and $y \in I \Rightarrow x \in I$.

An $\alpha$-ideal $I$ of $X$ is called closed, if $0 * x \in I \forall x \in X$.

A fuzzy set $\mu$ in a BF-algebra $X$ can be called as a fuzzy subalgebra of $X$, if it satisfies:

$$
\mu(x * y) \geq \mu(x) \wedge \mu(y) \text { for all } x, y \in X
$$

A fuzzy set $\mu$ in a BF-algebra $X$ can be called as a fuzzy ideal of $X$, if it satisfies:

$$
\begin{gathered}
\mu(0) \geq \mu(x) \text { for all } x \in X \\
\mu(x) \geq \mu(x * y) \wedge \mu(y) \text { for all } x, y \in X
\end{gathered}
$$


A fuzzy set $\mu$ in a BF-algebra $X$ can be called as a fuzzy $\alpha$-ideal of $X$, if it satisfies:

$$
\begin{gathered}
\mu(0) \geq \mu(0)(x) \text { for all } x \in X \\
\mu(x) \geq \mu((x * z) *(y * z)) \wedge \mu(y) \text { for all } x, y, z \in X
\end{gathered}
$$

Definition 2.4. [6] A function $f: X \rightarrow Y$ of BF-algebras is considered to be homomorphism of $X$, if

$$
f(x * y)=f(x) * f(y) \forall x, y \in X .
$$

Remark 2.5. If $f: X \rightarrow Y$ is a homomorphism on BF-algebras, $f\left(0_{X}\right)=0_{Y}$.

Definition 2.6. [6] A function $f: X \rightarrow Y$ of BF-algebras is said to be anti-homomorphism of $X$ if

$$
f(x * y)=f(y) * f(x) \forall x, y \in X .
$$

\subsection{Basic Results on Bipolar Valued Fuzzy Set}

Fuzzy sets are generally useful mathematical structures which represent a collection of objects whose boundary is vague. Several kinds of fuzzy set extensions are there in the fuzzy set theory. The examples are intuitionistic fuzzy sets, interval-valued fuzzy sets, vague sets, etc. This section starts with the definition of Bipolar Valued Fuzzy Set.

Definition 2.7. Let $X$ be a non empty set. A Bipolar Valued Fuzzy Set (BVFS) $B$ in $X$ is an object with the form

$$
B=\left\{\left(x ; \mu^{+}(x), v^{-}(x)\right) \mid x \in X\right\}
$$

where $\mu^{+}: X \rightarrow[0,1]$ and $v^{-}: X \rightarrow[-1,0]$ are mappings.

The positive membership degree $\mu^{+}(x)$ denotes the satisfaction degree of an element $x$ to the property corresponding to a bipolar valued fuzzy set $B=\left\{\left(x ; \mu^{+}(x), v^{-}(x)\right) \mid x \in X\right\}$ and the negative membership degree $v^{-}(x)$ denotes the satisfaction degree of an element $\mathrm{x}$ to some implicit counter-property corresponding to a bipolar valued fuzzy set $B=\left\{\left(x ; \mu^{+}(x), v^{-}(x)\right) \mid x \in X\right\}$. If $\mu^{+}(x) \neq 0$ and $v^{-}(x)=0, \quad x$ is regarded as possessing only positive satisfaction for $B=\left\{\left(x ; \mu^{+}(x), v^{-}(x)\right) \mid x \in X\right\}$. If $\mu^{+}(x)=0$ and $v^{-}(x) \neq 0$, it denotes that $x$ does not satisfy the property of $B=\left\{\left(x ; \mu^{+}(x), v^{-}(x)\right) \mid x \in X\right\}$ but somewhat satisfies the counter property of $B=\left\{\left(x ; \mu^{+}(x), v^{-}(x)\right) \mid x \in X\right\}$. It is possible for an element $x$ to be such that $\mu^{+}(x) \neq 0$ and $v^{-}(x) \neq 0$ when the membership function of the property overlaps that of its counter property over some portion of $X$. For the sake of simplicity, the symbol $B=\left(X ; \mu^{+}, v^{-}\right)$shall be used for the bipolar valued fuzzy set $B=\left\{\left(x ; \mu^{+}(x), v^{-}(x)\right) \mid x \in X\right\}$.

Definition 2.8. A BVFS $B$ in a set $X$ with the positive membership $\mu^{+}: X \rightarrow[0,1]$ and negative membership $v^{-}: X \rightarrow[0,1]$ is indicated to have Sup-Inf property, if for any subset $T$ of $X$, there exists $x_{0} \in T$ such that $\mu^{+}\left(x_{0}\right)=\sup _{t \in T} \mu^{+}(t)$ and $v^{-}\left(x_{0}\right)=\inf _{t \in T} v^{-}(t)$.

Definition 2.9. Let $f: X \rightarrow Y$ be a function and let $A=\left(X ; \mu_{A}^{+}, v_{A}^{-}\right)$and $B=\left(Y ; \mu_{B}^{+}, v_{B}^{-}\right)$be the bipolar valued fuzzy sets of $X$ and $Y$, respectively. Then, the image of $A$ under $f$ is defined as $f(A)=\left(Y ; \mu_{f(A)}^{+}, v_{f(A)}^{-}\right)$ such that

$$
\mu_{f(A)}^{+}(y)= \begin{cases}\sup _{z \in f^{-1}(y)} \mu_{A}^{+}(z) & \text { if } f^{-1}(y)=\{x: f(x)=y\} \neq \phi \\ 0 & \text { otherwise }\end{cases}
$$


and

$$
v_{f(A)}^{-}(y)= \begin{cases}\inf _{z \in f^{-1}(y)} v_{A}^{-}(z) & \text { if } f^{-1}(y)=\{x: f(x)=y\} \neq \phi \\ 0 & \text { otherwise }\end{cases}
$$

Definition 2.10. Let $f: X \rightarrow Y$ be a function and let $A=\left(X ; \mu_{A}^{+}, v_{A}^{-}\right)$and $B=\left(Y ; \mu_{B}^{+}, v_{B}^{-}\right)$be the bipolar valued fuzzy sets of $X$ and $Y$, respectively. Then, the inverse image of $B$ under $f$ is defined as

$$
f^{-1}(B)=\left(X ; \mu_{f^{-1}(B)}^{+}, v_{f^{-1}(B)}^{-}\right)
$$

such that $\mu_{f^{-1}(B)}^{+}(x)=\mu_{B}^{+}(f(x))$ and $v_{f^{-1}(B)}^{-}(x)=v_{B}^{-}(f(x)) \forall x \in X$.

\section{Bipolar Valued Fuzzy $\alpha$-Ideal}

In this section, Bipolar valued fuzzy $\alpha$-ideal of a BF-algebra is defined. It is also proved that any Bipolar valued fuzzy $\alpha$-ideal in $X$ is a Bipolar valued fuzzy BF-ideal and the sufficient condition is derived for the converse.

Definition 3.1. A BVFS $B=\left(X ; \mu^{+}, v^{-}\right)$in $X$ is called a bipolar valued fuzzy subalgebra of $X$, if it satisfies:

$$
\begin{gathered}
\mu^{+}(x * y) \geq \mu^{+}(x) \wedge \mu^{+}(y) \\
v^{-}(x * y) \leq v^{-}(x) \vee v^{-}(y) \quad \forall x, y \in X
\end{gathered}
$$

Definition 3.2. A BVFS $B=\left(X ; \mu^{+}, v^{-}\right)$in $X$ is called a bipolar valued fuzzy ideal (BVF-ideal) of $X$, if it satisfies:

$$
\begin{gathered}
\mu^{+}(0) \geq \mu^{+}(x) \text { and } v^{-}(0) \leq v^{-}(x) \\
\mu^{+}(x) \geq \mu^{+}(x * y) \wedge \mu^{+}(y) \\
v^{-}(x) \leq v^{-}(x * y) \vee v^{-}(y) \forall x, y \in X .
\end{gathered}
$$

Definition 3.3. A BVFS B in a BF-algebra $X$, is to be a Bipolar Valued Fuzzy Closed BF-ideal (BVFC-BFideal) of $X$, if

- $\mu^{+}(x) \geq \mu^{+}(x * y) \wedge \mu^{+}(y)$

- $v^{-}(x) \leq v^{-}(x * y) \vee v^{-}(y)$

- $\mu^{+}(0 * x) \geq \mu^{+}(x)$

- $v^{-}(0 * x) \leq v^{-}(x) \forall x, y \in X$.

Definition 3.4. A BVFS $A$ in a BF-algebra $X$ is called to be a Bipolar Valued Fuzzy $\alpha$-ideal (BVF- $\alpha$-ideal) of $X$, if

- $\mu_{A}^{+}(0) \geq \mu_{A}^{+}(x)$ and $v_{A}^{-}(0) \leq v_{A}^{-}(x)$

- $\mu_{A}^{+}(x) \geq \mu_{A}^{+}((x * z) *(y * z)) \wedge \mu_{A}^{+}(y)$

- $v_{A}^{-}(x) \leq v_{A}^{-}((x * z) *(y * z)) \vee v_{A}^{-}(y) \forall x, y, z \in X$.

Example 3.5. The BF-algebra $X=\{0,1,2,3\}$ is considered with the Cayley table as given below.

\begin{tabular}{lllll}
\hline$*$ & 0 & 1 & 2 & 3 \\
\hline 0 & 0 & 1 & 2 & 3 \\
1 & 1 & 0 & 3 & 2 \\
2 & 2 & 3 & 0 & 1 \\
3 & 3 & 2 & 1 & 0 \\
\hline
\end{tabular}

$A=\left\{\left\langle x, \mu_{A}^{+}(x), v_{A}^{-}(x)\right\rangle \mid x \in X\right\}$ is the BVFS of $X$ defined as 


$$
\mu_{A}^{+}(x)=\left\{\begin{array}{lr}
1 ; \quad x=0,1 \\
0.5 ; x=2,3
\end{array} \text { and } v_{A}^{-}(x)= \begin{cases}-1 ; & x=0,1 \\
-0.6 ; & x=2,3\end{cases}\right.
$$

is a BVF- $\alpha$-ideal of $X$.

Definition 3.6. A BVFS $A$ in a BF-algebra $X$ is considered to be a Bipolar valued fuzzy closed $\alpha$-ideal (BVFC- $\alpha$-ideal) of $X$, if

- $\mu_{A}^{+}(x) \geq \mu_{A}^{+}((x * z) *(y * z)) \wedge \mu_{A}^{+}(y)$

- $v_{A}^{-}(x) \leq v_{A}^{-}((x * z) *(y * z)) \vee v_{A}^{-}(y)$

- $\mu_{A}^{+}(0 * x) \geq \mu_{A}^{+}(x)$

- $v_{A}^{-}(0 * x) \leq v_{A}^{-}(x) \forall x, y, z \in X$.

Example 3.7. Consider the BF-algebra $X=\{0,1,2,3\}$ with the Cayley table given below.

\begin{tabular}{lllll}
\hline$*$ & 0 & 1 & 2 & 3 \\
\hline 0 & 0 & 1 & 2 & 3 \\
1 & 1 & 0 & 3 & 2 \\
2 & 2 & 3 & 0 & 1 \\
3 & 3 & 2 & 1 & 0 \\
\hline
\end{tabular}

$A=\left\{\left\langle x, \mu_{A}^{+}(x), v_{A}^{-}(x)\right\rangle \mid x \in X\right\}$ is the BVFS of $X$ defined as

$$
\mu_{A}^{+}(x)=\left\{\begin{array}{ll}
0.8 ; & x=0,1 \\
0.1 ; & x=2,3
\end{array} \text { and } v_{A}^{-}(x)= \begin{cases}-0.5 ; & x=0,1 \\
-0.1 ; & x=2,3\end{cases}\right.
$$

is a BVFC- $\alpha$-ideal of $X$.

Trivially, the following can be proved:

Proposition 3.8. Every BVFC- $\alpha$-ideal is a BVF- $\alpha$-ideal.

In general, the converse of the above proposition is not true from the following:

Example 3.9. Consider the BF-algebra $X=\{0,1,2,3\}$ with the Cayley table given below

\begin{tabular}{lllll}
\hline$*$ & 0 & 1 & 2 & 3 \\
\hline 0 & 0 & 3 & 0 & 1 \\
1 & 1 & 0 & 1 & 3 \\
2 & 2 & 3 & 0 & 1 \\
3 & 3 & 1 & 3 & 0 \\
\hline
\end{tabular}

$A=\left\{\left\langle x, \mu_{A}^{+}(x), v_{A}^{-}(x)\right\rangle \mid x \in X\right\}$ is the BVFS of $\mathrm{X}$ defined as

$$
\mu_{A}^{+}(x)=\left\{\begin{array}{ll}
0.6 ; & x=0,1 \\
0.2 ; & x=2,3
\end{array} \text { and } v_{A}^{-}(x)= \begin{cases}-0.7 ; & x=0,1 \\
-0.3 ; & x=2,3\end{cases}\right.
$$

is a BVF- $\alpha$-ideal of $X$ but not BVFC- $\alpha$-ideal.

Since $\mu_{A}^{+}(0 * 1)<\mu_{A}^{+}(1)$ and $v_{A}^{-}(0 * 1) \leq v_{A}^{-}(1)$.

Proposition 3.10. If $A$ is Bipolar valued fuzzy $\alpha$-ideal of $X$ with $x \leq y$ for any $x, y \in X$, then $\mu_{A}^{+}(x) \geq \mu_{A}^{+}(y)$ and $v_{A}^{-}(x) \leq v_{A}^{-}(y)$. That is $\mu_{A}^{+}$is order-reversing and $v_{A}^{-}$is order-preserving.

Proof: Let $x, y, z \in X$ such that $x \leq y \leq z$.

Then, by the partial ordering if $\leq$ is defined in $X, \quad x * y=0$ and $y * z=0$. 
Thus, $\quad \mu_{A}^{+}(x) \geq \mu_{A}^{+}((x * y) *(y * z)) \wedge \mu_{A}^{+}(y)$

$\geq \mu_{A}^{+}\left((0 * 0) \wedge \mu_{A}^{+}(y)\right)=\mu_{A}^{+}(0) \wedge \mu_{A}^{+}(y)=\mu_{A}^{+}(y)$.

And

$$
\begin{aligned}
v_{A}^{-}(x) & \leq v_{A}^{-}((x * y) *(y * z)) \vee v_{A}^{-}(y) \\
& \leq v_{A}^{-}\left((0 * 0) \vee v_{A}^{-}(y)\right)=v_{A}^{-}(0) \vee v_{A}^{-}(y)=v_{A}^{-}(y) .
\end{aligned}
$$

It completes the proof.

Theorem 3.11. If $A$ is BVFC- $\alpha$-ideal of $X$, then the sets $J=\left\{x \in X ; \mu_{A}^{+}(x)=\mu_{A}^{+}(0)\right\}$ and $K=\left\{x \in X ; v_{A}^{-}(x)=v_{A}^{-}(0)\right\}$ are $\alpha$-ideals of $X$.

Proof: Clearly, $0 \in J$ and $0 \in K$. Hence, $J \neq \phi$ and $K \neq \phi$.

Let $(x * y) *(y * z) \in J$ and $y \in J$.

$\Rightarrow \mu_{A}^{+}((x * y) *(y * z))=\mu_{A}^{+}(y)=0$

$\Rightarrow \mu_{A}^{+}(x) \geq \mu_{A}^{+}((x * y) *(y * z)) \wedge \mu_{A}^{+}(y)=0 \wedge 0=0$.

But $\mu_{A}^{+}(0) \geq \mu_{A}^{+}(x) \Rightarrow \mu_{A}^{+}(x)=0$.

Hence, $J$ is an $\alpha$-ideal of $X$. Similarly, it can be proved that $K$ is an $\alpha$-ideal of $X$.

Theorem 3.12. Any BVF- $\alpha$-ideal of $X$ is a Bipolar valued fuzzy BF-ideal of $X$.

Proof: It is trivial by putting $z=0$ in the definition of BVF- $\alpha$-ideal.

The converse of the above theorem may not be true.

Now, a sufficient condition is derived for a Bipolar valued fuzzy BF-ideal to be a BVF- $\alpha$-ideal as follows:

Theorem 3.13. Let $A$ be a BVF-BF-ideal of $X$. If $\mu_{A}^{+}(x * y) \geq \mu_{A}^{+}((x * z) *(y * z))$ and $v_{A}^{-}(x * y) \leq v_{A}^{-}((x * z) *(y * z)) \forall x, y, z \in X$, then $A$ is BVF- $\alpha$-ideal of $X$.

Proof: Let $A$ be a Bipolar valued fuzzy BF-ideal of $X$ and assign $\quad x, y, z \in X$.

So, we have $\mu_{A}^{+}(0) \geq \mu_{A}^{+}(x)$ and $v_{A}^{-}(0) \leq v_{A}^{-}(x)$.

Then,

$$
\mu_{A}^{+}(x) \geq \mu_{A}^{+}(x * y) \wedge \mu_{A}^{+}(y) \geq \mu_{A}^{+}((x * z) *(y * z)) \wedge \mu_{A}^{+}(y)
$$

and

$$
v_{A}^{-}(x) \leq v_{A}^{-}(x * y) \vee v_{A}^{-}(y) \leq v_{A}^{-}((x * z) *(y * z)) \vee v_{A}^{-}(y) .
$$

Hence, $A$ is BVF- $\alpha$-ideal of $X$.

Theorem 3.14. The intersection of any two Bipolar valued fuzzy $\alpha$-ideals of $X$ is also a Bipolar valued fuzzy $\alpha$-ideal.

Proof: Let A and B be any two Bipolar valued fuzzy $\alpha$-ideals of $X$.

Let $A=\left\{\left\langle x, \mu_{A}^{+}(x), v_{A}^{-}(x)\right\rangle \mid x \in X\right\}$ and $B=\left\{\left\langle x, \mu_{B}^{+}(x), v_{B}^{-}(x)\right\rangle \mid x \in X\right\}$.

Consider

$$
C=A \cap B=\left\{\left\langle x, \mu_{C}^{+}(x), v_{C}^{-}(x)\right\rangle \mid x \in X\right\}
$$

where $\mu_{C}^{+}(x)=\mu_{A}^{+}(x) \wedge \mu_{B}^{+}(x)$ and $v_{C}^{-}(x)=v_{A}^{-}(x) \vee v_{B}^{-}(x)$.

Let $x, y \in X$.

Now, $\mu_{C}^{+}(0)=\mu_{A}^{+}(0) \wedge \mu_{B}^{+}(0) \geq \mu_{A}^{+}(x) \wedge \mu_{B}^{+}(x)=\mu_{C}^{+}(x)$ and

$$
\begin{gathered}
v_{C}^{-}(0)=v_{A}^{-}(0) \vee v_{B}^{-}(0) \leq v_{A}^{-}(x) \vee v_{B}^{-}(x)=v_{C}^{-}(x) . \\
\mu_{C}^{+}(x)=\mu_{A}^{+}(x) \wedge \mu_{B}^{+}(x) \\
\geq\left(\mu_{A}^{+}((x * z) *(y * z)) \wedge \mu_{A}^{+}(y)\right) \wedge\left(\mu_{B}^{+}((x * z) *(y * z)) \wedge \mu_{B}^{+}(y)\right) \\
=\left(\mu_{A}^{+}((x * z) *(y * z)) \wedge \mu_{B}^{+}((x * z) *(y * z))\right) \wedge\left(\mu_{A}^{+}(y) \wedge \mu_{B}^{+}(y)\right) \\
=\mu_{C}^{+}((x * z) *(y * z)) \wedge \mu_{C}^{+}(y)
\end{gathered}
$$

Similarly, $v_{C}^{-}(x) \leq v_{C}^{-}((x * z) *(y * z)) \vee v_{C}^{-}(y)$, and it completes the proof. 
The above theorem can be generalized as follows.

Theorem 3.15.The intersection of a family of Bipolar valued fuzzy $\alpha$-ideals of $X$ is a Bipolar valued fuzzy $\alpha$-ideal of $X$.

The following can be analogously proved.

Theorem 3.16. Intersection of any two Bipolar valued fuzzy closed $\alpha$-ideal of $X$ is also a Bipolar valued fuzzy closed $\alpha$-ideal of $X$. Hence, the intersection of a family of Bipolar valued fuzzy closed $\alpha$-ideal of $X$ is also a Bipolar valued fuzzy closed $\alpha$-ideal of $X$.

Remark 3.17. $B=\left(X ; \mu^{+}, v^{-}\right)$is a BVFS defined on any universe $X$, if and only if $\mu^{+}$and $-v^{-}$are the fuzzy subsets of $X$.

Theorem 3.18. A BVFS $B=\left(X ; \mu^{+}, v^{-}\right)$is a BVF- $\alpha$-ideal of $X$, if and only if the fuzzy subsets $\mu^{+}$and $-v^{-}$are fuzzy $\alpha$-ideals of $X$.

Proof: Let $B=\left(X ; \mu^{+}, v^{-}\right)$be a BVF- $\alpha$-ideal of $X$.

Further, clearly $\mu^{+}$is a fuzzy $\alpha$-ideal of $X$.

Also $v^{-}(0) \leq v^{-}(x)$ and $v_{A}^{-}(x) \leq v_{A}^{-}((x * z) *(y * z)) \vee v_{A}^{-}(y) \forall x, y, z \in X$.

$$
\Rightarrow-v^{-}(0) \geq-v^{-}(x) \text { and }-v^{-}(x) \geq-v_{A}^{-}((x * z) *(y * z)) \wedge-v_{A}^{-}(y)
$$

Therefore, $-v^{-}$is a fuzzy $\alpha$-ideal of $X$

Conversely, assume $\mu^{+}$and $-v^{-}$are fuzzy $\alpha$-ideals of $X$.

It is enough to prove that, $v^{-}(0) \leq v^{-}(x)$ and $v_{A}^{-}(x) \leq v_{A}^{-}((x * z) *(y * z)) \vee v_{A}^{-}(y) \forall x, y, z \in X$.

For, $-v^{-}(0) \geq-v^{-}(x)$ and $-v^{-}(x) \geq-v_{A}^{-}((x * z) *(y * z)) \wedge-v_{A}^{-}(y)$.

$$
\Rightarrow v^{-}(0) \leq v^{-}(x) \text { and } v_{A}^{-}(x) \leq v_{A}^{-}((x * z) *(y * z)) \vee v_{A}^{-}(y) .
$$

It fulfills the proof.

The following can be obtained using this theorem.

Theorem 3.19. A BVFS $B=\left\{\left\langle x, \mu_{A}^{+}(x), v_{A}^{-}(x)\right\rangle \mid x \in X\right\}$ is a BVF- $\alpha$-ideal of $X$, if and only if

- $\square B=\left(X ; \mu^{+},-\mu^{+}\right)$and

- $\diamond B=\left(X ;-v^{-}, v^{-}\right)$are also BVF- $\alpha$-ideals of $X$.

Proof: $A=\left\{\left\langle x, \mu_{A}^{+}(x), v_{A}^{-}(x)\right\rangle \mid x \in X\right\}$ is a Bipolar valued fuzzy $\alpha$-ideal of $X$, if and only if, the fuzzy subsets $\mu^{+}$and $-v^{-}$are fuzzy $\alpha$-ideals of $X$ by the theorem 3.18 .

That is, if and only if, $\square B$ and $\diamond B$ are also Bipolar valued fuzzy $\alpha$-ideal of $X$ by the definition of $\square B$ and $\diamond B$.

The following is analogously true.

Theorem 3.20. A BVFS $B=\left\{\left\langle x, \mu_{A}^{+}(x), v_{A}^{-}(x)\right\rangle \mid x \in X\right\}$ is a BVFC- $\alpha$-ideal of $X$ if and only if

- $\square B=\left(X ; \mu^{+},-\mu^{+}\right)$and

- $\diamond B=\left(X ;-v^{-}, v^{-}\right)$are also BVFC- $\alpha$-ideals of $X$.

\section{Homomorphism on Bipolar Valued Fuzzy $\alpha$-Ideal}

Here, the image and pre-image of Bipolar valued fuzzy $\alpha$-ideals under the action of homomorphism and antihomomorphism on BF-algebras are discussed.

Theorem 4.1. Let $f$ be a homomorphism from BF-algebras $X$ onto $Y$. A be a bipolar valued fuzzy $\alpha$-ideal of $X$ with Sup-Inf property. Then, the image of $A, \quad f(A)=\left\{\left\langle y, \mu_{f(A)}^{+}, v_{f(A)}^{-}(y)\right\rangle \mid y \in Y\right\}$ is a bipolar valued fuzzy $\alpha$ ideal of $Y$.

Proof: Let $a, b, c \in Y$ with $x_{0} \in f^{-1}(a), y_{0} \in f^{-1}(b)$ and $z_{0} \in f^{-1}(c)$ such that 


$$
\mu_{A}^{+}\left(x_{0}\right)=\sup _{t \in f^{-1}(a)} \mu_{A}^{+}(t) ; \mu_{A}^{+}\left(y_{0}\right)=\sup _{t \in f^{-1}(b)} \mu_{A}^{+}(t) ; \mu_{A}^{+}\left(z_{0}\right)=\sup _{t \in f^{-1}(c)} \mu_{A}^{+}(t)
$$

and

$$
v_{A}^{-}\left(x_{0}\right)=\inf _{t \in f^{-1}(a)} v_{A}^{-}(t) ; v_{A}^{-}\left(y_{0}\right)=\inf _{t \in f^{-1}(b)} v_{A}^{-}(t) ; v_{A}^{-}\left(z_{0}\right)=\inf _{t \in f^{-1}(c)} v_{A}^{-}(t)
$$

Now, by the definitions 2.8, 2.9 and 2.4, the following is framed

$$
\mu_{f(A)}^{+}(0)=\sup _{t \in f^{-1}(0)} \mu_{A}^{+}(t) \geq \mu_{A}^{+}(0) \geq \mu_{A}^{+}\left(x_{0}\right)=\sup _{t \in f^{-1}(a)} \mu_{A}^{+}(t)=\mu_{f(A)}^{+}(a)
$$

and

$$
\begin{aligned}
& v_{f(A)}^{-}(0)=\inf _{t \in f^{-1}(0)} v_{A}^{-}(t) \leq v_{A}^{-}(0) \leq v_{A}^{-}\left(x_{0}\right)=\inf _{t \in f^{-1}(a)} v_{A}^{-}(t)=v_{f(A)}^{-}(a) \\
& \mu_{f(A)}^{+}((a * c) *(b * c)) \wedge \mu_{f(A)}^{+}(b)=\sup _{t \in f^{-1}((a * c) *(b * c))} \mu_{A}^{+}(t) \wedge \sup _{t \in f^{-1}(b)} \mu_{A}^{+}(t) \\
& \leq \mu_{A}^{+}\left(\left(x_{0} * z_{0}\right) *\left(y_{0} * z_{0}\right)\right) \wedge \mu_{A}^{+}\left(y_{0}\right) \\
& \leq \mu_{A}^{+}\left(x_{0}\right)= \sup _{t \in f^{-1}(a)} \mu_{A}^{+}(t)=\mu_{f(A)}^{+}(a) \\
& v_{f(A)}^{-}((a * c) *(b * c)) \vee v_{f(A)}^{-}(b)=\inf _{t \in f^{-1}((a * c) *(b * c))} v_{A}^{-}(t) \vee \inf _{t \in f^{-1}(b)} v_{A}^{-}(t) \\
& \geq v_{A}^{-}\left(\left(x_{0} * z_{0}\right) *\left(y_{0} * z_{0}\right)\right) \vee v_{A}^{-}\left(y_{0}\right) \\
& \geq v_{A}^{-}\left(x_{0}\right)=\inf _{t \in f^{-1}(a)} v_{A}^{-}(t)=v_{f(A)}^{-}(a)
\end{aligned}
$$

Now,

Hence, the image $f(A)$ is a bipolar valued fuzzy $\alpha$-ideal of $Y$.

Theorem 4.2. Let $f$ be a homomorphism from BF-algebras $X$ onto $Y$ and $A$ be a Bipolar valued fuzzy closed $\alpha$-ideal of $X$ with Sup-Inf property. Then, the image of $A, f(A)=\left\{\left\langle y, \mu_{f(A)}^{+}(y), v_{f(A)}^{-}(y)\right\rangle \mid y \in Y\right\}$ is a bipolar valued fuzzy closed $\alpha$-ideal of $Y$.

Proof: Let $x \in Y$ with $x_{0} \in f^{-1}(x)$ such that

$$
\mu_{A}^{+}\left(x_{0}\right)=\sup _{t \in f^{-1}(x)} \mu_{A}^{+}(t) ; v_{A}^{-}\left(x_{0}\right)=\inf _{t \in f^{-1}(x)} v_{A}^{-}(t) .
$$

Then, we have

$$
\begin{gathered}
\mu_{f(A)}^{+}(x)=\sup _{t \in f^{-1}(x)} \mu_{A}^{+}(t) \leq \mu_{A}^{+}\left(x_{0}\right) \leq \mu_{A}^{+}\left(0 * x_{0}\right)=\sup _{t \in f^{-1}(0 * x)} \mu_{A}^{+}(t)=\mu_{f(A)}^{+}(0 * x) \\
v_{f(A)}^{-}(x)=\inf _{t \in f^{-1}(x)} v_{A}^{-}(t) \geq v_{A}^{-}\left(x_{0}\right) \geq v_{A}^{-}\left(0 * x_{0}\right)=\inf _{t \in f^{-1}(0 * x)} v_{A}^{-}(t)=v_{f(A)}^{-}(0 * x)
\end{gathered}
$$

Hence, by the above theorem, the image $f(A)$ is considered as a Bipolar valued fuzzy closed $\alpha$-ideal of $Y$.

Theorem 4.3. Let $f$ be a homomorphism from BF-algebras $X$ onto $Y$ and $B$ be a bipolar valued fuzzy $\alpha$-ideal of $Y$. Then, the inverse image of $B, f^{-1}(B)$ is a bipolar valued fuzzy $\alpha$-ideal of $X$.

Proof: Let $x, y \in X$.

Now, it is clear that

$$
\begin{aligned}
& \mu_{f^{-1}(B)}^{+}(0)=\mu_{B}^{+}(f(0)) \geq \mu_{B}^{+}(f(x))=\mu_{f^{-1}(B)}^{+}(x) \text { and } v_{f^{-1}(B)}^{-}(0)=v_{B}^{-}(f(0)) \leq v_{B}^{-}(f(x))=v_{f^{-1}(B)}^{-}(x) \\
& \text { Then, } \\
& \mu_{f^{-1}(B)}^{+}(x)=\mu_{B}^{+}(f(x)) \geq \mu_{B}^{+}((f(x) * f(z)) *(f(y) * f(z))) \wedge \mu_{B}^{+}(f(y)) \\
& =\mu_{f^{-1}(B)}^{+}((x * z) *(y * z)) \wedge \mu_{f^{-1}(B)}^{+}(y) \\
& \text { Also } v_{f^{-1}(B)}^{-}(x)=v_{B}^{-}(f(x)) \leq v_{B}^{-}((f(x) * f(z)) *(f(y) * f(z))) \vee v_{B}^{-}(f(y)) \\
& =v_{f^{-1}(B)}^{-}((x * z) *(y * z)) \vee v_{f^{-1}(B)}^{-}(y)
\end{aligned}
$$


Then, the inverse image of $B, f^{-1}(B)$ is a bipolar valued fuzzy $\alpha$-ideal of $X$.

Theorem 4.4. Let $f$ be a homomorphism from BF-algebras $X$ onto $Y$ and $B$ be a Bipolar valued fuzzy closed $\alpha$-ideal of $Y$. Then the inverse image of $B, f^{-1}(B)$ is a Bipolar valued fuzzy closed $\alpha$-ideal of $X$.

Proof: Let $x \in X$. Then, we have

$$
\begin{aligned}
& \mu_{f^{-1}(B)}^{+}(0 * x)=\mu_{B}^{+}(f(0 * x))=\mu_{B}^{+}(f(0) * f(x)) \geq \mu_{B}^{+}(f(x))=\mu_{f^{-1}(B)}^{+}(x) \\
& v_{f^{-1}(B)}^{-}(0 * x)=v_{B}^{-}(f(0 * x))=v_{B}^{-}(f(0) * f(x)) \leq v_{B}^{-}(f(x))=v_{f^{-1}(B)}^{-}(x) .
\end{aligned}
$$

Hence, through the above theorem, the inverse image $f^{-1}(B)$ becomes a Bipolar valued fuzzy closed $\alpha$ ideal of $X$.

In the same way, the following can be proved.

Theorem 4.5. Let $f$ be an anti-homomorphism from $X$ onto $Y$ and A be a bipolar valued fuzzy $\alpha$-ideal of $X$ with Sup-Inf property. Then, the image of $A, f(A)$ is a bipolar valued fuzzy $\alpha$-ideal of $Y$.

Theorem 4.6. Let $f$ be an anti-homomorphism from $X$ onto $Y$ and $B$ be a bipolar valued fuzzy $\alpha$-ideal of $Y$. Then, the inverse image of $B, f^{-1}(B)$ is a bipolar valued fuzzy $\alpha$-ideal of $X$.

Theorem 4.7. Let $f$ be an anti-homomorphism from $X$ onto $Y$ and $A$ be a bipolar valued fuzzy closed $\alpha$-ideal of $X$ with Sup-Inf property. Then, the image of $A, f(A)$ is a bipolar valued fuzzy closed $\alpha$-ideal of $Y$.

Theorem 4.8. Let $f$ be an anti-homomorphism from $X$ onto $Y$ and $B$ be a bipolar valued fuzzy closed $\alpha$-ideal of $Y$. Then, the inverse image of $B, f^{-1}(B)$ is a bipolar valued fuzzy closed $\alpha$-ideal of $X$.

\section{Conclusion}

From the preliminaries of this research work, Bipolar valued fuzzy sets of various researchers are analyzed. Especially, for the present work stated in this paper, an investigation on the Bipolar valued fuzzy $\alpha$-ideals of BF-algebras has been carried out. From the investigation, several interesting results are observed. As a result, the research has been focused on this way and all the possible ways are found out to prove this strategy. The surprising point is that in [7] Andrzej Walendziak, theorem 2.11 says that the structure of BF algebra becomes a BG-algebra and the proof is followed directly from the definition. Hence, it is concluded that all the results prove here for BF-algebras can directly be carried over to BG-algebras.

\section{Acknowledgements}

The authors would like to thank the anonymous reviewers for their insightful and constructive comments and suggestions that have led to an improved version of this paper.

\section{References}

[1] Zadeh, L.A. (1965) Fuzzy Sets. Inform Control, 8, 338-353. http://dx.doi.org/10.1016/S0019-9958(65)90241-X

[2] Lee, K.M. (2000) Bipolar Valued Fuzzy Sets and Their Applications. Proceedings of International Conference on Intelligent Technologies, Bangkok, 307-312.

[3] Lee, K.M. (2004) Comparison of Interval-Valued Fuzzy Sets, Intuitionistic Fuzzy Sets and Bipolar Valued Fuzzy Sets. Fuzzy Logic Intelligent Systems, 14, 125-129. http://dx.doi.org/10.5391/jkiis.2004.14.2.125

[4] Farhat Nisar, (2011) Bipolar-Valued Fuzzy K-subalgebras. World Applied Sciences Journal, 14, 1914-1919.

[5] Muralikrishna, P. and Chandramouleeswaran, M. (2012) A Note on BF-Algebras with Intuitionistic L-Fuzzy p-Ideals. Scientia Magna, 8, 98-110.

[6] Jun, J.B. and Park, C.H. (2009) Filters of BCH-Algebras Based on Bipolar-Valued Fuzzy Sets. International Mathematical Forum, 4, 631-643.

[7] Walendziak, A. (2007) On BF-Algebras. Mathematica Slovaca, 57. 


\section{Submit or recommend next manuscript to SCIRP and we will provide best service for you:}

Accepting pre-submission inquiries through Email, Facebook, LinkedIn, Twitter, etc.

A wide selection of journals (inclusive of 9 subjects, more than 200 journals)

Providing 24-hour high-quality service

User-friendly online submission system

Fair and swift peer-review system

Efficient typesetting and proofreading procedure

Display of the result of downloads and visits, as well as the number of cited articles

Maximum dissemination of your research work

Submit your manuscript at: http://papersubmission.scirp.org/ 